\title{
Barley varieties registered in the Czech Republic after harvest 2018
}

\author{
Vratislav Psota ${ }^{1 *}$, Olga Dvořáčková2, Milan Nečas², Markéta Musilová ${ }^{1}$ \\ 1 Research Institute of Brewing and Malting, Mostecká 971/7, \\ 61400 Brno, Czech Republic \\ 2 Central Institute for Supervising and Testing in Agriculture, \\ National Plant Variety Office, Hroznová 2, CZ-656 06 Brno _ Corresponding author: psota@beerresearch.cz
}

\begin{abstract}
This study presents the results of malting quality and agronomic characters obtained within three-year testing of barley varieties for the registration in the Czech Republic. New spring barley malting varieties rich in extract, i.e. Cosmopolitan, Ismena, Klarinette, Laureate, LG Aurus, and Runner were registered after the 2018 harvest. The extract of the varieties Cosmopolitan, Laureate, and LG Aurus was higher than $83 \%$. The values of Kolbach index, diastatic power and apparent final attenuation were at the above-average to optimal level. The level of cytolytic modification was at the above-average to optimal level with the exception of the variety Runner which exhibited higher $\beta$-glucan content in wort (192 mg/l). In addition, non-malting varieties of winter six-row barley Azrah, Impala, Journey, Laurin, SU Jule, and a hybrid variety SU Hylona were registered.
\end{abstract}

Key words: barley, variety, malting quality

\section{Introduction}

In the Czech Republic, new barley varieties are registered under Act 219/2003 on the basis of a three-year testing, which is carried out according to the Methodology of Barley Utility Value Testing (Dvořáčková, 2016). The tests for the registration of new varieties include monitoring the utility value, i.e. yield and other yield characteristics, resistance to diseases, lodging and traits characterizing malt quality. The quality of malt samples was evaluated on the basis of the characteristics given in the malting quality index (Psota and Kosař, 2002).

The present study evaluates the technological and agronomic quality of the spring barley malting varieties Cosmopolitan, Ismena, Klarinette, Laureate, LG Aurus, and Runner, which completed the trials for the registration in 2018. In addition, the non-malting varieties of winter six-row barley Azrah, Impala, Journey, Laurin, SU Jule and the hybrid variety SU Hylona were also registered. The utility value of the non-malting varieties is given only in the table without verbal description.

\section{Material and Methods}

Malting quality of the studied spring barley varieties was assessed based on the analysis of 12 malt samples made in the micromalting plant. Seed samples were delivered by the National Plant Variety Office of the Central Institute for Supervising and Testing in Agriculture (CISTA) in Brno in 2016-2018.

Malting quality was assessed mainly on the basis of the parameters making up the malting quality index (further only MQI) (Psota and Kosař, 2002). Further, we studied whether the variety provided clear wort. In the set of the varieties under study, none met the criteria for recommendation and production of the beer with the protected geographical indication "České pivo" (European Commission, 2008).

\subsection{Determination of technological quality}

\subsubsection{Selection of the testing sites}

Grain samples of the tested varieties were collected annu- 
ally from four testing sites in which the standard varieties exhibited the optimal content of nitrogenous substances (10.2-11.2\%). Thus it was secured that the technological parameters determined were not negatively affected by a low or, on the contrary, an unfavorably high content of nitrogenous substances in grain. In the course of the three-year testing cycle, a total of 12 values were obtained from each studied parameter.

\subsubsection{Varieties under study}

The list of all barley varieties registered after harvest 2018 is given in Table 1. For comparison of the utility value of the advanced breeding lines with the utility value of the already registered varieties, CISTA includes in the registration trials the standard varieties which, after previous negotiations with applicants and representatives of foreign companies, proceed together with the tested varieties throughout the three-year testing procedure.

The set of the standard registered varieties always includes at least one variety which completed the registration procedure with the recommendation for production of beer with the protected geographical indication "České pivo", further varieties with the malting quality for the production of other types of beers, and at least one non-malting variety which achieves high yield of grain. The standard varieties serve for the assessment of yield while the other parameters, such as resistance to diseases or resistance to lodging are assessed according to scales (Dvořáčková, 2016). The MQI parameters are assessed within fixed scopes.

\subsubsection{Malting and malt analysis}

Samples of barley varieties (500 grams) were malted in the KVM (Uničov, Czech Republic) automatic micromalting equipment. The RIBM always uses the same regime of steeping, germination and kilning for varietal testing. The conditions and procedure of malting are given in Table 2 . Sieving fractions over $2.5 \mathrm{~mm}$ are used for the micromalting test. Malt analyses are given in Table 3 including references to the methods used (EBC Analysis committee, 2010, MEBAK, 2011, Baxter and O’Farrell, 1983).

\subsection{Determination of agronomical characteristics}

Every year, the agronomical characteristics were assessed according to the methods of CISTA (Dvořáčková,
2016) in spring barley varieties (Table 4) in 14 testing localities and in winter barley varieties (Table 5) in 8 testing localities.

Table 1 Assortment of registered barley varieties after harvest 2018

\begin{tabular}{l|l|}
\multicolumn{1}{c|}{ Variety / Code } & \multicolumn{1}{c|}{ Maintainer / Agent in the CR } \\
\cline { 2 - 3 } $\begin{array}{l}\text { Cosmopolitan } \\
\text { SJ 152037 }\end{array}$ & \begin{tabular}{l}
\multicolumn{1}{c|}{ malting varieties } \\
Sejet Planteforaedling I/S (DNK) \\
SELGEN, a.s.
\end{tabular} \\
\hline $\begin{array}{l}\text { Ismena } \\
\text { NORD 14/2403 }\end{array}$ & $\begin{array}{l}\text { NORDSAAT Saatzucht GmbH (DEU) } \\
\text { SAATEN - UNION CZ s.r.o. }\end{array}$ \\
\hline $\begin{array}{l}\text { Klarinette } \\
\text { SC 101-12A }\end{array}$ & $\begin{array}{l}\text { SECOBRA Recherches (FRA) } \\
\text { SOUFFLET AGRO a.s. }\end{array}$ \\
\hline $\begin{array}{l}\text { Laureate } \\
\text { SY 412-328 }\end{array}$ & $\begin{array}{l}\text { Syngenta Participations AG (GBR) } \\
\text { B O R, s.r.o. }\end{array}$ \\
\hline $\begin{array}{l}\text { LG Aurus } \\
\text { LGBHE3427A }\end{array}$ & $\begin{array}{l}\text { Limagrain Europe (FRA) } \\
\text { Limagrain Central Europe Cereals, s.r.o. }\end{array}$ \\
\hline $\begin{array}{l}\text { Runner } \\
\text { NORD 14/2534 }\end{array}$ & $\begin{array}{l}\text { NORDSAAT Saatzucht GmbH (DEU) } \\
\text { SAATEN - UNION CZ s.r.o. }\end{array}$ \\
\hline \multicolumn{1}{|c|}{ winter barley } & non-malting varieties \\
\hline $\begin{array}{l}\text { Azrah } \\
\text { STRG 432/09 }\end{array}$ & $\begin{array}{l}\text { Saatzucht Streng - Engelen GmbH \& Co.KG (DEU) } \\
\text { B O R, s.r.o. }\end{array}$ \\
\hline $\begin{array}{l}\text { Impala } \\
\text { LEU 43408 }\end{array}$ & $\begin{array}{l}\text { Deutsche Saatveredelung AG (DEU) } \\
\text { Ing. Marian Špunar }\end{array}$ \\
\hline $\begin{array}{l}\text { Journey } \\
\text { KW 6-451 }\end{array}$ & $\begin{array}{l}\text { KWS LOCHOW GMBH (DEU) } \\
\text { SOUFFLET AGRO a.s. }\end{array}$ \\
\hline $\begin{array}{l}\text { Laurin } \\
\text { NORD 11002/8 }\end{array}$ & $\begin{array}{l}\text { NORDSAAT Saatzucht GmbH (DEU) } \\
\text { SELGEN, a.s. }\end{array}$ \\
\hline $\begin{array}{l}\text { SU Hylona } \\
\text { DEH 13/1807 }\end{array}$ & $\begin{array}{l}\text { ASUR PLANT BREEDING s.a.s. (FRA) } \\
\text { SAATEN - UNION CZ s.r.o. }\end{array}$ \\
\hline $\begin{array}{l}\text { SU Jule } \\
\text { BE 2008108012 }\end{array}$ & $\begin{array}{l}\text { W. von Borries-Eckendorf GmbH \& Co. KG (DEU) } \\
\text { Ing. Marian Špunar }\end{array}$ \\
\hline
\end{tabular}

Agricultural characters of varieties include:

- yield of grain at standard 14\% moisture content in the non-treated (without the use of fungicides) and treated (two treatments with fungicides) variants of growing. Yield of grain and yield of grain over $2.5 \mathrm{~mm}$ in spring barley in terms of the response of the varieties to the soil and weather conditions and suitability of the use of grain for malting are assessed within the testing area (maize, sugar-beet, cereal, and potato). In winter barley, all the areas are assessed jointly.

- agronomical data (time to heading, maturity, number of productive stems, length of plants, resistance to lodging). - resistance to diseases (powdery mildew of barley (Blumeria graminis), leaf rust of barley (Puccinia hordei), net blotch of barley (Pyrenophora teres), scald of barley (Rhynchosporium secalis), scab of barley (Fusarium graminearum, F culmorum, Microdochium nivale etc.), physiological leaf spots of barley (non-specific leaf spots). - quality parameters of the grain (thousand grain weight and sievings over $2.5 \mathrm{~mm}$ ). 
Table 2 Conditions and schedule of malting

\begin{tabular}{|c|c|c|c|c|c|}
\hline & Time & $\begin{array}{l}\text { Temperature of } \\
\text { ingoing air }\end{array}$ & $\begin{array}{c}\text { Temperature of } \\
\text { outgoing air }\end{array}$ & $\begin{array}{l}\text { Fan } \\
\text { speed }\end{array}$ & $\begin{array}{c}\text { Air } \\
\text { recirculation }\end{array}$ \\
\hline & h & ${ }^{\circ} \mathrm{C}$ & ${ }^{\circ} \mathrm{C}$ & $\%$ & $\%$ \\
\hline \multicolumn{6}{|l|}{ Steeping } \\
\hline Wet period & 5.0 & & \multirow{6}{*}{14.0} & & \\
\hline Dry period & 19.0 & & & & \\
\hline Wet period & 4.0 & & & & \\
\hline Dry period & 20.0 & & & & \\
\hline Wet period & \multirow{2}{*}{$* 24.0$} & & & & \\
\hline Dry period & & & & & \\
\hline Germination & 72.0 & & 14.0 & & \\
\hline \multirow{8}{*}{ Kilning } & 1.0 & 14.0 to 55.0 & 14.0 to 25.0 & \multirow{8}{*}{70} & 0 \\
\hline & 11.0 & 55.0 & 25.0 to 35.0 & & 0 \\
\hline & 1.0 & 55.0 to 60.0 & 40.0 to 45.0 & & 40 \\
\hline & 1.0 & 60.0 to 65.0 & 45.0 to 50.0 & & 40 \\
\hline & 2.0 & 65.0 to 70.0 & 50,0 to 55.0 & & 40 \\
\hline & 1.0 & 70.0 to 75.0 & 55.0 to 65.0 & & 40 \\
\hline & 1.0 & 75.0 to 80.0 & 65.0 to 78.0 & & 80 \\
\hline & 4.0 & 80 & 78 & & 80 \\
\hline
\end{tabular}

* Water content was adjusted to $45 \%$ by steeping or spraying.

Table 3 Barley grain and malt analyses (2016-2018)

\begin{tabular}{|c|c|c|c|c|c|c|c|c|c|c|c|c|}
\hline Methods & Units & References & 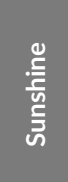 & 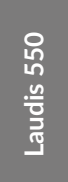 & 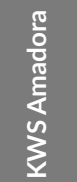 & 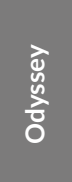 & 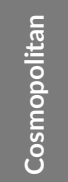 & हूँ & 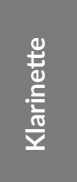 & 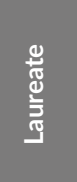 & $\sum_{0}^{\frac{n}{2}}$ & 㐫 \\
\hline & & & $\mathrm{S}$ & S & S & $S$ & & & & & & \\
\hline $\begin{array}{l}\text { Protein content } \\
\text { of barley (factor 6.25) }\end{array}$ & $\%$ & EBC 2010 & 11.1 & 11.1 & 10.2 & 10.4 & 10.4 & 10.4 & 10.6 & 10.3 & 10.5 & 10.2 \\
\hline $\begin{array}{l}\text { Starch content } \\
\text { of barley }\end{array}$ & $\%$ & NIR & 63.6 & 63.8 & 64.2 & 64.6 & 64.6 & 64.2 & 64.4 & 64.2 & 64.5 & 64.3 \\
\hline Degree of steeping 1 & $\%$ & & 32.4 & 31.6 & 31.1 & 31.5 & 31.8 & 32.6 & 32.5 & 32.1 & 33.7 & 33.6 \\
\hline Degree of steeping 2 & $\%$ & & 40.1 & 39.1 & 38.8 & 39.2 & 39.2 & 40.1 & 40.0 & 39.7 & 41.5 & 41.3 \\
\hline Malt yield d. m. & $\%$ & Briggs 1998 & 90.9 & 91.2 & 91.2 & 91.9 & 91.8 & 92.0 & 91.5 & 91.9 & 91.2 & 91.6 \\
\hline Respiration losses d. m. & $\%$ & Briggs 1998 & 4.6 & 4.3 & 4.5 & 4.3 & 3.9 & 4.2 & 4.2 & 3.8 & 4.4 & 4.2 \\
\hline Rootlet losses d. m. & $\%$ & Briggs 1998 & 4.5 & 4.5 & 4.3 & 3.8 & 4.3 & 3.8 & 4.2 & 4.3 & 4.4 & 4.2 \\
\hline $\begin{array}{l}\text { Extract of malt, con- } \\
\text { gress mash }\end{array}$ & $\%$ & EBC 2010 & 82.7 & 82.4 & 83.9 & 82.9 & 83.1 & 82.4 & 82.9 & 83.9 & 83.5 & 82.5 \\
\hline $\begin{array}{l}\text { Mash method accord- } \\
\text { ing to Hartong and } \\
\text { Kretschmer VZ } 45^{\circ} \mathrm{C}\end{array}$ & $\%$ & МЕВАК 2011 & 43.6 & 38.6 & 50.1 & 42.0 & 48.3 & 44.0 & 39.0 & 45.8 & 44.6 & 44.9 \\
\hline Kolbach index & $\%$ & EBC 2010 & 49.9 & 43.7 & 52.6 & 46.7 & 48.4 & 49.3 & 43.2 & 50.6 & 47.5 & 46.1 \\
\hline Diastatic power & WK & EBC 2010 & 443 & 346 & 397 & 327 & 350 & 275 & 279 & 333 & 341 & 279 \\
\hline $\begin{array}{l}\text { Final attenuation } \\
\text { of laboratory wort }\end{array}$ & $\%$ & EBC 2010 & 82.9 & 80.5 & 83.5 & 82.3 & 82.9 & 81.9 & 81.2 & 82.3 & 82.3 & 82.0 \\
\hline Friability & $\%$ & EBC 2010 & 92 & 85 & 98 & 89 & 92 & 87 & 84 & 90 & 88 & 84 \\
\hline $\begin{array}{l}\text { High molecular weight } \\
\text { beta-glucan content } \\
\text { of malt, SFA }\end{array}$ & $\mathrm{mg} / \mathrm{l}$ & EBC 2010 & 49 & 153 & 36 & 144 & 106 & 166 & 119 & 145 & 156 & 192 \\
\hline
\end{tabular}




\begin{tabular}{|c|c|c|c|c|c|c|c|c|c|c|c|c|}
\hline Methods & Units & References & 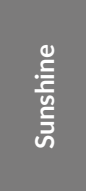 & 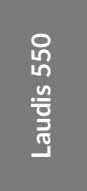 & 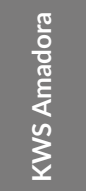 & $\begin{array}{l}\text { वे } \\
\text { पू } \\
\text { ते }\end{array}$ & 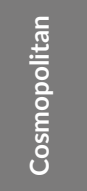 & $\begin{array}{l}\frac{\mathbb{2}}{\mathrm{d}} \\
\frac{\mathrm{E}}{\mathrm{n}}\end{array}$ & 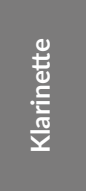 & 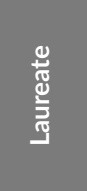 & 氙 & 远 \\
\hline & & & $S$ & $S$ & $S$ & $S$ & & & & & & \\
\hline $\begin{array}{l}\text { Protein content of malt } \\
\text { (factor } 6.25 \text { ) }\end{array}$ & $\%$ & EBC 2010 & 10.1 & 10.1 & 9.2 & 9.4 & 9.3 & 9.4 & 9.6 & 9.2 & 9.4 & 9.2 \\
\hline $\begin{array}{l}\text { Total nitrogen of malt, } \\
\text { Kjeldahl method }\end{array}$ & $\%$ & EBC 2010 & 1.61 & 1.61 & 1.47 & 1.51 & 1.50 & 1.51 & 1.54 & 1.47 & 1.51 & 1.47 \\
\hline $\begin{array}{l}\text { Soluble nitrogen of } \\
\text { wort, Kjeldahl method }\end{array}$ & $\mathrm{mg} / \mathrm{l}$ & EBC 2010 & 899 & 788 & 860 & 778 & 809 & 831 & 743 & 832 & 801 & 761 \\
\hline $\begin{array}{l}\text { Soluble nitrogen of } \\
\text { malt, Kjeldahl method }\end{array}$ & $\mathrm{mg} / 100 \mathrm{~g}$ & EBC 2010 & 802 & 703 & 769 & 693 & 722 & 740 & 663 & 743 & 717 & 679 \\
\hline $\begin{array}{l}\text { Soluble nitrogen of } \\
\text { malt, Kjeldahl method }\end{array}$ & $\%$ & EBC 2010 & 0.802 & 0.703 & 0.769 & 0.693 & 0.722 & 0.740 & 0.663 & 0.743 & 0.717 & 0.679 \\
\hline $\begin{array}{l}\text { Viscosity of laboratory } \\
\text { wort from malt }\end{array}$ & $\mathrm{mPa} . \mathrm{s}$ & EBC 2010 & 1.451 & 1.491 & 1.454 & 1.466 & 1.458 & 1.479 & 1.485 & 1.467 & 1.465 & 1.475 \\
\hline $\begin{array}{l}\text { Colour of malt, visual } \\
\text { method }\end{array}$ & EBC & EBC 2010 & 3.22 & 2.90 & 3.55 & 3.18 & 3.57 & 3.58 & 2.80 & 3.88 & 3.26 & 3.38 \\
\hline Saccharification time & $\min$ & EBC 2010 & 10 & 11 & 10 & 10 & 10 & 10 & 11 & 10 & 10 & 11 \\
\hline Glassy corns & $\%$ & EBC 2010 & 0.0 & 0.1 & 0.0 & 0.0 & 0.1 & 0.1 & 0.2 & 0.1 & 0.0 & 0.1 \\
\hline $\begin{array}{l}\text { Partly unmodified } \\
\text { grains }\end{array}$ & $\%$ & EBC 2010 & 0.3 & 1.6 & 0.2 & 1.2 & 0.5 & 1.4 & 2.3 & 1.3 & 1.1 & 2.1 \\
\hline $\begin{array}{l}\text { Homogeneity } \\
\text { (by friabilimeter) }\end{array}$ & $\%$ & $\begin{array}{l}\text { Baxter, O'Farrell } \\
1983\end{array}$ & 99.7 & 98.4 & 99.9 & 98.8 & 99.5 & 98.7 & 97.7 & 98.7 & 98.9 & 97.9 \\
\hline $\begin{array}{l}\text { Appearance (clarity) } \\
\text { of wort }\end{array}$ & & MEBAK 2011 & 1.08 & 1.00 & 1.08 & 1.00 & 1.17 & 1.33 & 1.08 & 1.17 & 1.00 & 1.00 \\
\hline Haze of wort $\left(90^{\circ}\right)$ & EBC & EBC 2010 & 0.89 & 0.90 & 1.10 & 0.69 & 1.32 & 2.30 & 1.15 & 1.45 & 0.77 & 0.68 \\
\hline Haze of wort $\left(12^{\circ}\right)$ & EBC & EBC 2010 & 1.08 & 1.05 & 1.14 & 0.82 & 1.29 & 2.34 & 1.22 & 1.49 & 1.00 & 0.84 \\
\hline $\begin{array}{l}\text { Total polyphenols } \\
\text { in wort }\end{array}$ & $\mathrm{mg} / \mathrm{l}$ & EBC 2010 & 70.7 & 64.5 & 91.7 & 82.3 & 83.9 & 79.5 & 69.3 & 77.5 & 86.7 & 74.8 \\
\hline Free amino nitrogen & $\mathrm{mg} / \mathrm{l}$ & EBC 2010 & 198 & 168 & 207 & 172 & 186 & 186 & 157 & 186 & 182 & 164 \\
\hline Free amino nitrogen & $\mathrm{mg} / 100 \mathrm{~g}$ & EBC 2010 & 153 & 129 & 172 & 153 & 148 & 154 & 125 & 126 & 148 & 141 \\
\hline
\end{tabular}

$\mathrm{S}=$ standard varieties

Notes to some parameters given in the table:

- Wort clarity is assessed as follows: 1 = clear, 2 = weakly opalizing, 3 = opalizing, 4 = cloudy.

- Malt yield in dry matter (\%) = weight of deculmed malt in d.m. (weight of barley in d.m. / 100).

- Respiration losses (\%) = 100 - (malt yield in d.m. + rootlet losses).

- Rootlet losses (\%) = 100- weight of deculmed malt / (weight of non-deculmed malt / 100)

- Total malting losses in d.m. (\%) = [(weight of barley in d.m. - weight of deculmed malt in d.m.) * 100] / weight of barley in d.m. (Briggs, 1998).

\section{Results}

The micromalting tests with the varieties Cosmopolitan, Ismena, Klarinette, Laureate, LG Aurus, and Runner were conducted in 2016-2018 within testing for registration. The highest malt yield (92.0\%) was exhibited by the variety Ismena. In the set of the varieties under study, no variety met the criteria for recommendation and production of beer with the protected geographical indication "České pivo" (European Commission, 2008).

The malts obtained from the spring barley varieties exhibited a high extract content (82.4-83.9\%). The content of nitrogenous substances in non-malted grain moved within the optimal values (10.2-10.6\%).
A high content of soluble nitrogen (over $800 \mathrm{mg} / \mathrm{l}$ ) was exhibited by the varieties Laureate ( $832 \mathrm{mg} / \mathrm{l})$, Ismena (831 mg/l), Cosmopolitan (809 mg/l), and LG Aurus $(801 \mathrm{mg} / \mathrm{l})$. Proteolytic modification characterized by Kolbach index ranged from 43.2-50.6\%. Proteolytic modification of the varieties Laureate and Ismena was high $(50.6 \%$ and $49.3 \%)$. Free amino nitrogen was in the range of $157-186 \mathrm{mg} / \mathrm{l}$ and formed $22.2 \%$ of soluble nitrogen (39.0-48.3\%). Relative extract at $45{ }^{\circ} \mathrm{C}$ characterizing the activity of namely cytolytic and proteolytic enzymes was at the optimal level (39.0-48.3\%). The activity of amylolytic enzymes hydrolyzing starch, namely $\beta$-amylase, was at the optimal level. The value of diastatic power ranged from 275-350 WK units. 
Table 4 Important agricultural properties (2016-2018)

\begin{tabular}{|c|c|c|c|c|c|c|c|c|c|c|c|c|c|}
\hline Variety & $\begin{array}{l}\frac{\vec{t}}{\mathrm{~d}} \\
\text { 壳 }\end{array}$ & 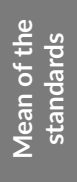 & $\begin{array}{l}\frac{\pi}{0} \\
\frac{0}{0} \\
\frac{1}{8} \\
\frac{1}{4} \\
\frac{1}{3}\end{array}$ & 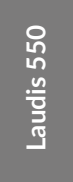 & 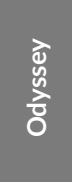 & 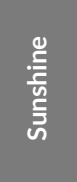 & $\begin{array}{l}\frac{\pi}{\frac{N}{0}} \\
\frac{\mathrm{C}}{\mathrm{N}}\end{array}$ & 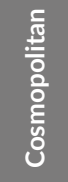 & $\begin{array}{l}\text { 듬 } \\
\text { E् }\end{array}$ & 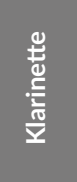 & $\begin{array}{l}\frac{y}{\mathbb{d}} \\
\frac{d}{2} \\
\mathbb{J}\end{array}$ & $\begin{array}{l}\frac{n}{2} \\
\frac{3}{7} \\
0\end{array}$ & 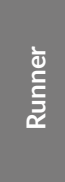 \\
\hline Grain yield in (t/ha) & & & S & $\mathbf{S}$ & S & $\mathbf{S}$ & S & & & & & & \\
\hline maize growing region & $\begin{array}{l}\mathbf{N} \\
\mathbf{T}\end{array}$ & $\begin{array}{l}6.07 \\
6.53\end{array}$ & $\begin{array}{l}6.09 \\
6.58\end{array}$ & $\begin{array}{l}5.94 \\
6.54\end{array}$ & $\begin{array}{l}5.92 \\
6.16\end{array}$ & $\begin{array}{l}5.95 \\
6.42\end{array}$ & $\begin{array}{l}6.42 \\
6.94\end{array}$ & $\begin{array}{l}6.13 \\
6.57\end{array}$ & $\begin{array}{l}6.23 \\
6.71\end{array}$ & $\begin{array}{l}6.25 \\
6.69\end{array}$ & $\begin{array}{l}6.28 \\
6.66\end{array}$ & $\begin{array}{l}6.37 \\
6.71\end{array}$ & $\begin{array}{l}6.41 \\
6.94\end{array}$ \\
\hline $\begin{array}{l}\text { sugar beet and cereal } \\
\text { growing regions }\end{array}$ & $\begin{array}{l}\mathrm{N} \\
\mathrm{T}\end{array}$ & $\begin{array}{l}7.48 \\
7.87\end{array}$ & $\begin{array}{l}7.39 \\
7.91\end{array}$ & $\begin{array}{l}7.41 \\
7.57\end{array}$ & $\begin{array}{l}7.53 \\
7.95\end{array}$ & $\begin{array}{l}7.38 \\
7.75\end{array}$ & $\begin{array}{l}7.69 \\
8.17\end{array}$ & $\begin{array}{l}7.77 \\
8.35\end{array}$ & $\begin{array}{l}7.91 \\
8.37\end{array}$ & $\begin{array}{l}8.01 \\
8.22\end{array}$ & $\begin{array}{l}8.04 \\
8.38\end{array}$ & $\begin{array}{l}7.68 \\
8.02\end{array}$ & $\begin{array}{l}7.80 \\
8.34\end{array}$ \\
\hline $\begin{array}{l}\text { potato and forage } \\
\text { growing regions }\end{array}$ & $\begin{array}{l}\mathrm{N} \\
\mathrm{T}\end{array}$ & $\begin{array}{l}6.51 \\
7.38\end{array}$ & $\begin{array}{l}6.73 \\
7.84\end{array}$ & $\begin{array}{l}6.29 \\
7.00\end{array}$ & $\begin{array}{l}6.78 \\
7.29\end{array}$ & $\begin{array}{l}6.17 \\
6.99\end{array}$ & $\begin{array}{l}6.59 \\
7.76\end{array}$ & $\begin{array}{l}7.10 \\
8.06\end{array}$ & $\begin{array}{l}6.96 \\
8.01\end{array}$ & $\begin{array}{l}7.28 \\
7.62\end{array}$ & $\begin{array}{l}7.01 \\
7.60\end{array}$ & $\begin{array}{l}7.01 \\
7.40\end{array}$ & $\begin{array}{l}7.10 \\
8.21\end{array}$ \\
\hline \multicolumn{14}{|c|}{ Grain over $2.5 \mathrm{~mm}$ (t/ha) } \\
\hline maize growing region & $\begin{array}{l}\mathrm{N} \\
\mathrm{T}\end{array}$ & $\begin{array}{l}4.81 \\
5.49\end{array}$ & $\begin{array}{l}4.53 \\
5.37\end{array}$ & $\begin{array}{l}4.80 \\
5.46\end{array}$ & $\begin{array}{l}4.91 \\
5.31\end{array}$ & $\begin{array}{l}4.78 \\
5.60\end{array}$ & $\begin{array}{l}5.03 \\
5.70\end{array}$ & $\begin{array}{l}4.91 \\
5.52\end{array}$ & $\begin{array}{l}4.58 \\
5.58\end{array}$ & $\begin{array}{l}4.82 \\
5.44\end{array}$ & $\begin{array}{l}5.32 \\
5.91\end{array}$ & $\begin{array}{l}4.87 \\
5.46\end{array}$ & $\begin{array}{l}4.82 \\
5.60\end{array}$ \\
\hline $\begin{array}{l}\text { sugar-beet and cereal } \\
\text { growing regions }\end{array}$ & $\begin{array}{l}\mathrm{N} \\
\mathrm{T}\end{array}$ & $\begin{array}{l}7.01 \\
7.52\end{array}$ & $\begin{array}{l}6.97 \\
7.63\end{array}$ & $\begin{array}{l}6.97 \\
7.21\end{array}$ & $\begin{array}{l}7.14 \\
7.66\end{array}$ & $\begin{array}{l}7.07 \\
7.51\end{array}$ & $\begin{array}{l}6.88 \\
7.60\end{array}$ & $\begin{array}{l}7.38 \\
8.02\end{array}$ & $\begin{array}{l}7.25 \\
7.81\end{array}$ & $\begin{array}{l}7.46 \\
7.68\end{array}$ & $\begin{array}{l}7.79 \\
8.18\end{array}$ & $\begin{array}{l}7.28 \\
7.67\end{array}$ & $\begin{array}{l}7.09 \\
7.78\end{array}$ \\
\hline $\begin{array}{l}\text { potato and forage } \\
\text { growing regions }\end{array}$ & $\begin{array}{l}\mathrm{N} \\
\mathrm{T}\end{array}$ & $\begin{array}{l}6.22 \\
7.21\end{array}$ & $\begin{array}{l}6.43 \\
7.67\end{array}$ & $\begin{array}{l}5.99 \\
6.82\end{array}$ & $\begin{array}{l}6.53 \\
7.16\end{array}$ & $\begin{array}{l}6.00 \\
6.85\end{array}$ & $\begin{array}{l}6.17 \\
7.54\end{array}$ & $\begin{array}{l}6.78 \\
7.90\end{array}$ & $\begin{array}{l}6.58 \\
7.79\end{array}$ & $\begin{array}{l}6.96 \\
7.42\end{array}$ & $\begin{array}{l}6.83 \\
7.44\end{array}$ & $\begin{array}{l}6.81 \\
7.27\end{array}$ & $\begin{array}{l}6.70 \\
7.93\end{array}$ \\
\hline \multicolumn{14}{|c|}{ Agronomic data } \\
\hline straw length (cm) & & & 68 & 71 & 71 & 72 & 71 & 67 & 69 & 70 & 69 & 71 & 67 \\
\hline earliness of ripening** & & & 110 & 110 & 111 & 111 & 110 & 111 & 109 & 110 & 111 & 110 & 109 \\
\hline standing power (lodging & sistan & & 6.1 & 6.7 & 5.5 & 7.0 & 7.2 & 6.7 & 7.3 & 6.8 & 6.0 & 5.6 & 7.0 \\
\hline \multicolumn{14}{|c|}{ Resistance to diseases } \\
\hline $\begin{array}{l}\text { powdery mildew of barl } \\
\text { (Blumeria graminis) }\end{array}$ & & & 8.9 & 8.9 & 9.0 & 5.8 & 6.7 & 8.9 & 8.9 & 8.3 & 8.9 & 8.8 & 9.0 \\
\hline $\begin{array}{l}\text { leaf rust of barley } \\
\text { (Puccinia hordei) }\end{array}$ & & & 5.1 & 6.5 & 6.0 & 7.1 & 7.1 & 6.0 & 5.5 & 6.5 & 6.5 & 7.0 & 6.0 \\
\hline $\begin{array}{l}\text { net blotch of barley } \\
\text { (Pyrenophora teres) }\end{array}$ & & & 6.8 & 6.5 & 6.8 & 7.0 & 5.9 & 6.3 & 6.8 & 7.0 & 7.2 & 6.9 & 6.7 \\
\hline $\begin{array}{l}\text { scald of barley } \\
\text { (Rhynchosporium secalis) }\end{array}$ & & & 7.2 & 7.5 & 8.1 & 8.0 & 6.2 & 8.2 & 8.6 & 7.7 & 7.6 & 6.5 & 6.6 \\
\hline $\begin{array}{l}\text { scab of barley (Fusarium } \\
\text { F. culmorum, Microdochi }\end{array}$ & $\begin{array}{l}\text { aminec } \\
\text { nivale }\end{array}$ & & 6.9 & 7.3 & 7.0 & 5.6 & 6.3 & 6.2 & 6.3 & 6.4 & 7.2 & 7.2 & 6.0 \\
\hline $\begin{array}{l}\text { physiological leaf spots } \\
\text { (non-specific leaf spots) }\end{array}$ & barley & & 8.2 & 6.0 & 8.3 & 7.9 & 8.1 & 8.4 & 8.3 & 8.5 & 8.5 & 8.0 & 8.5 \\
\hline \multicolumn{14}{|c|}{ Grain quality } \\
\hline 1000 grain weight $(\mathrm{g})$ & & & 48 & 46 & 48 & 49 & 46 & 49 & 49 & 47 & 50 & 52 & 50 \\
\hline sieving fractions over 2 & $\mathrm{~nm}(\%$ & & 92 & 91 & 93 & 93 & 89 & 92 & 89 & 90 & 94 & 92 & 89 \\
\hline
\end{tabular}

$S=$ standard varieties

Point evaluation: 1 = fully lodging, fully attacked $\quad 9$ = non lodging, resistant to diseases

Weight of 1000 grains relates to sieving fractions over $2.0 \mathrm{~mm}$ at $14 \%$ humidity.

* days from sowing to cropping maturity (time from $1 / 1$ to the maturity day)

Intensity: $N$ - non treated with fungicides and morphoregulators $T$ - treated with fungicides and morphoregulators

Degradation of cell walls was easy (friability over 85\%) in the varieties Cosmopolitan, Laureate, LG Aurus, and Ismena. Favorable values of $\beta$-glucan content in wort were recorded in the varieties Cosmopolitan (106 mg/l) and Klarinete $(119 \mathrm{mg} / \mathrm{l})$.

The wort color in the studied varieties was from 3.2 to $3.8 \mathrm{EBC}$ units and it strongly reflected the level of pro- teolytic modification. The varieties LG Aurus and Runner always provided clear wort. Only the variety Ismena provided wort haze determined by nephelometer at the level of 2.3 EBC units. In the other varieties this level was significantly lower.

Malt of the variety Cosmopolitan, which was bred in Denmark, provided an above average extract yield 
Table 5 Significant agricultural properties (2016-2018)

\begin{tabular}{|c|c|c|c|c|c|c|c|c|c|c|}
\hline \multirow[t]{3}{*}{ Variety } & \multirow[t]{3}{*}{ 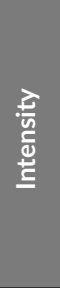 } & \multirow{3}{*}{ 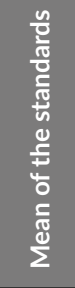 } & 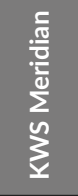 & $\sum_{i=1}^{n}$ & $\frac{\frac{\pi}{\pi / N}}{\frac{10}{4}}$ & $\begin{array}{l}\frac{\pi}{\pi} \\
\text { 을 }\end{array}$ & $\begin{array}{l}\text { ఫे } \\
\text { है } \\
\text { 윽 }\end{array}$ & 惡 & $\begin{array}{l}\frac{\varrho}{\Xi} \\
\frac{2}{n}\end{array}$ & $\begin{array}{l}\text { ¿ } \\
\text { 을 } \\
\text { 졸 } \\
\text { 己 }\end{array}$ \\
\hline & & & \multicolumn{8}{|c|}{ 6-row } \\
\hline & & & S & S & & & & & & \\
\hline \multirow{2}{*}{ grain yield (t/ha) } & $\mathrm{N}$ & 7.62 & 7.69 & 7.55 & 7.52 & 7.59 & 7.94 & 7.83 & 7.68 & 7.92 \\
\hline & $\mathbf{T}$ & 9.10 & 9.20 & 9.01 & 9.25 & 9.28 & 9.14 & 9.27 & 9.27 & 9.45 \\
\hline \multicolumn{11}{|c|}{ Agronomic data } \\
\hline straw length $(\mathrm{cm})$ & & & 97 & 107 & 96 & 99 & 98 & 100 & 101 & 108 \\
\hline earliness of ripening* & & & 183 & 184 & 183 & 185 & 184 & 184 & 184 & 184 \\
\hline standing power (lodging resistance) & & & 6.3 & 7.7 & 7.7 & 7.4 & 7.5 & 7.6 & 7.7 & 6.2 \\
\hline \multicolumn{11}{|c|}{ Resistance to diseases } \\
\hline \multicolumn{3}{|l|}{ powdery mildew of barley (Blumeria graminis) } & 6.9 & 7.5 & 6.0 & 7.6 & 7.3 & 7.3 & 5.9 & 6.8 \\
\hline \multicolumn{3}{|l|}{ leaf rust of barley (Puccinia hordei) } & 7.1 & 7.4 & 6.4 & 7.2 & 7.1 & 7.4 & 6.7 & 7.2 \\
\hline \multicolumn{3}{|l|}{ net blotch of barley (Pyrenophora teres) } & 6.8 & 6.3 & 7 & 6.7 & 6.4 & 6.7 & 6.5 & 6.7 \\
\hline \multicolumn{3}{|l|}{ scald of barley (Rhynchosporium secalis) } & 7.8 & 7.0 & 7.5 & 7.8 & 7.5 & 7.6 & 7.5 & 8.0 \\
\hline \multicolumn{3}{|c|}{$\begin{array}{l}\text { scab of barley (Fusarium graminearum, F. culmorum, Microdochium } \\
\text { nivale etc.) }\end{array}$} & 7.8 & 7.5 & 7.4 & 7.5 & 7.7 & 7.2 & 8.1 & 8.2 \\
\hline \multicolumn{3}{|c|}{ physiological leaf spots of barley (non-specific leaf spots) } & 6.9 & 7.4 & 6.9 & 7.4 & 6.1 & 6.9 & 7.2 & 7.0 \\
\hline \multicolumn{11}{|c|}{ Mechanical properties (grain quality) } \\
\hline \multicolumn{3}{|l|}{1000 grain weight $(\mathrm{g})$} & 44 & 47 & 46 & 42 & 44 & 47 & 48 & 41 \\
\hline \multicolumn{3}{|l|}{ sieving fractions over $2.5 \mathrm{~mm}(\%)$} & 84 & 83 & 83 & 74 & 84 & 89 & 82 & 70 \\
\hline
\end{tabular}

$S=$ standard varieties

Point evaluation: 1 = fully lodging, fully attacked

9 = non lodging, resistant to diseases

Weight of 1000 grains relates to sieving fractions over $2.0 \mathrm{~mm}$ at $14 \%$ humidity.

* days from sowing to cropping maturity (time from $1 / 1$ to the maturity day)

Intensity: $\mathrm{N}$ - non treated with fungicides and morphoregulators $T$ - treated with fungicides and morphoregulators

(83.1\%) at the optimal content of nitrogenous substances in non-malted grain (10.4\%). Proteolytic modification was optimal (Kolbach index was around the value of $48.4 \%$ ). The wort exhibited high values of soluble nitrogen $(809 \mathrm{mg} / \mathrm{l})$. Free amino nitrogen was at the level of $186 \mathrm{mg} / \mathrm{l}$ and formed $23.0 \%$ of soluble nitrogen. Amylolytic modification was high (diastatic power moved around the value of $350 \mathrm{WK}$ units). Degradation of cell walls was at the level of $92 \%$ and $\beta$-glucan content in wort moved on average around $106 \mathrm{mg} / \mathrm{l}$. The variety provided optimal wort quality, apparent final attenuation ranged on average around $82.9 \%$. In most cases, the variety provided clear wort. Wort color intensity was above average (3.6 EBC un.). Considering the values of the above technological parameters, the variety Cosmopolitan has a very good malting quality with the point evaluation 9 (8.7).

It was recorded in the Common Catalogue of Varieties of Agricultural Plant Species (European Commission, 2019) and it is admitted in Denmark and Great Britain.
Cosmopolitan is a mid-early malting variety. The plants are mid high to low, the variety is mid resistant to lodging and mid resistant to stem breaking. It has medium sized grain, portion of sieving fractions above $2.5 \mathrm{~mm}$ is medium high. It is resistant to powdery mildew of barley, medium resistant to leaf rust of barley, medium resistant to net blotch of barley, resistant to leaf scald and medium resistant to scab of barley. Yield of sieving fractions above $2.5 \mathrm{~mm}$ in the treated variant growing in the sugar-beet and cereal and potato regions is very high, in the untreated variant growing in the potato region high to very high, in the untreated variant growing in the sugar-beet and cereal regions high, in both variants growing in the maize region it is medium high.

The malt of the variety Ismena, which was bred in Germany, provided an above average extract yield $(82.4 \%)$ at the optimal content of nitrogenous substances in non-malted grain (10.4\%). Proteolytic modification was above average (Kolbach index moved around the value of 
49.3\%). Wort exhibited high values of soluble nitrogen $(831 \mathrm{mg} / \mathrm{l})$. Free amino nitrogen was at the medium level of $186 \mathrm{mg} / \mathrm{l}$ and formed $22.4 \%$ of soluble nitrogen. Amylolytic modification was above average (diastatic power moved around the value of $275 \mathrm{WK}$ units). Degradation of cell walls was at the level of $87 \%$ and $\beta$-glucan content in wort moved on average around $166 \mathrm{mg} / \mathrm{l}$. The variety provided optimal wort quality, apparent final attenuation ranged on average around $81.9 \%$. In most cases, the variety gave clear wort. Wort color was above average (3.7 EBC units). Considering the values of the studied technological parameters, the variety Ismena has very good malting quality with the point evaluation 7 (6.7).

It was recorded in the Common Catalogue of Varieties of Agricultural Plant Species (European Commission, 2019) and it is admitted in Poland.

Ismena is a mid-early to early malting variety. The plants are mid high to low, the variety is mid resistant to lodging and mid resistant to stem breaking. It has medium sized grain, portion of sieving portions above $2.5 \mathrm{~mm}$ is medium high to low. It is resistant to powdery mildew of barley, medium to less resistant to leaf rust of barley, medium resistant to net blotch of barley, resistant to leaf scald, and medium resistant to scab of barley. Yield of sieving fractions above $2.5 \mathrm{~mm}$ in the treated variant growing in the potato region is high to very high, in both variants growing in the sugar-beet and cereal regions and in the untreated variant growing medium high to high, in the treated variant growing in the maize region medium high, and low in the untreated variant growing in the maize region.

The malt of the German variety Klarinette was rich in extract (82.9\%) at the optimal content of nitrogenous substances in non-malted grain (10.6\%). Proteolytic modification was at the optimal level. The wort exhibited soluble nitrogen values at the level of $743 \mathrm{mg} / \mathrm{l}$ ). Free amino nitrogen was at the low level of $157 \mathrm{mg} / \mathrm{l}$ and formed $21.1 \%$ of soluble nitrogen. Amylolytic modification was above average (diastatic power moved around the value of $279 \mathrm{WK}$ units). Cytolytic modification was optimal. Degradation of cell walls was at the level of $84 \%$ and $\beta$-glucan content in wort moved on average around $119 \mathrm{mg} / \mathrm{l}$. The variety provided above average wort quality, apparent final attenuation moved on average around $81.2 \%$ ). In most cases, the variety gave clear wort. The color of the wort was medium to light (2.8 EBC units). In terms of the values of the studied technological parameters, the variety Klarinette has very good malting quality with the point evaluation 7 (7.5).

Klarinette is a mid-early malting variety. Mid high plants, the variety is mid resistant to lodging and mid resistant to stem breaking. It has medium big to small grain, the portion of sieving fractions above $2.5 \mathrm{~mm}$ is medium high. It is resistant to powdery mildew of barley, medium resistant to leaf rust of barley, medium resistant to net blotch of barley, medium resistant to leaf scald, medium resistant to scab of barley. Yield of sieving fractions above $2.5 \mathrm{~mm}$ in the untreated variant growing in the potato regions is very high, in the untreated variant growing in the sugar-beet and cereal regions high, in the treated variant growing in the sugar-beet, cereal and potato regions and in the untreated variant growing in the maize region medium high, in the treated variant growing in the maize region it is medium high to low.

Malt of the variety Laureate, which was bred in Great Britain, was rich in extract (83.9\%) at the optimal content of nitrogenous substances in non-malted grain at the level of $10.3 \%$. Proteolytic modification was high (50.6\%). The wort exhibited high values of soluble nitrogen at the level of $832 \mathrm{mg} / \mathrm{l}$. Free amino nitrogen was at the medium level of $186 \mathrm{mg} / \mathrm{l}$ and formed $22.3 \%$ of soluble nitrogen. Amylolytic modification was high (diastatic power $333 \mathrm{WK}$ units). Cytolytic modification was above average to optimal. Degradation of cell walls was at the level of $90 \%$ and $\beta$-glucan content in wort moved on average only around $145 \mathrm{mg} / \mathrm{l}$. The variety provided wort with a favorable composition, apparent final attenuation moved on average around $82.3 \%$. In most cases, the variety gave clear wort. Wort color was above average (3.9 EBC units). Considering the values achieved in the technological parameters, the variety Laureate has a very good malting quality with the point evaluation 7 (7.5).

The variety was recorded in the Common Catalogue of Varieties of Agricultural Plant Species (European Commission, 2019) and is admitted in Germany, Estonia, France, Lithuania, Latvia, the Netherlands, Austria and the United Kingdom. In 2019 it was also registered in the Slovak Republic (Psota et al., 2019).

Laureate is a mid-early malting variety. The plants are mid high to low, the variety is mid resistant to lodging and mid resistant to stem breaking. It has medium big grain, the portion of sieving fractions above $2.5 \mathrm{~mm}$ is high. It is resistant to powdery mildew of barley, medium resistant to leaf rust of barley, medium resistant to net blotch of barley, medium resistant to resistant to leaf scald, and medium resistant to scab of barley. Yield of sieving fractions above $2.5 \mathrm{~mm}$ in both variants growing in the maize, sugar-beet and cereal regions and in the untreated variant of growing in the potato region is very high, in the treated variant growing in the potato region it is medium high.

The malt of the Czech variety LG Aurus was rich in extract $(83.5 \%)$ at the optimal content of nitrogenous substances in non-malted grain $(10.5 \%)$. Proteolytic modification was at the optimal level. The wort exhibited 
high values of soluble nitrogen $(801 \mathrm{mg} / \mathrm{l})$. Free amino nitrogen was at the medium level of $182 \mathrm{mg} / \mathrm{l}$ and formed $22.7 \%$ of soluble nitrogen. Amylolytic modification was high (diastatic power moved around the value of $341 \mathrm{WK}$ units). Cytolytic modification was optimal. Degradation of cell walls was at the level of $88 \%$ and $\beta$-glucan content in wort moved on average around $156 \mathrm{mg} / \mathrm{l}$. The variety provided very favorable wort quality (apparent final attenuation ranged on average around $82.3 \%$ ). The variety always provided clear wort. Wort color was above average (3.3 EBC units). Considering the values of the technological parameters under study, the variety LG Aurus has very good malting quality with the point evaluation 8 (8.1).

In 2019 the variety was registered in the Slovak Republic (Psota et al., 2019). LG Aurus is a mid-early to early malting variety. The plants are mid high, the variety is mid too less resistant to lodging and mid resistant to stem breaking. Big grain, portion of sieving fractions above $2.5 \mathrm{~mm}$ is medium high. Resistant to powdery mildew of barley, medium resistant leaf rust of barley, medium resistant to net blotch of barley, medium resistant to leaf scald and medium resistant to scab of barley. Yield of sieving fractions above $2.5 \mathrm{~mm}$ in the untreated variant growing in the potato region is high to very high, in the untreated variant growing in the sugar-beet and cereal regions medium high to high, in the treated variant growing in the potato region medium high, in the untreated variant growing in the maize region and in the treated variant in the sugar-beet, cereal and potato regions medium high, in the treated variant in the maize region it is medium high to low.

The German variety Runner provided malt with above average values of extract yield (82.5\%) at the optimal content of nitrogenous substances in non-malted grain at the level of 10.2\%). Proteolytic modification was optimal (Kolbach index moved around the value of $46.1 \%)$. The wort exhibited soluble nitrogen values at the level of $761 \mathrm{mg} / \mathrm{l}$. Free amino nitrogen was at the level of $164 \mathrm{mg} / \mathrm{l}$ and formed $21.5 \%$ of soluble nitrogen. Amylolytic modification was above average (diastatic power moved around the value of 279 WK units). Cytolytic modification was average. Degradation of cell walls was at the level of $84 \%$ and $\beta$-glucan content in wort moved on average around $192 \mathrm{mg} / \mathrm{l}$. Within the tests, the variety achieved optimal values of apparent final attenuation $(82.0 \%)$ and always provided clear wort. Wort color was above average (3.4 EBC units). Considering the values of the technological parameters, the variety Runner has very good malting quality with the point evaluation 7 (6.7).

The variety Runner was recorded in the Common Catalogue of Varieties of Agricultural Plant Species (European Commission, 2019) and is admitted in Poland.
Runner is a mid-early to early malting variety. The plants are mid high to low, the variety is mid resistant to lodging and mid resistant to stem breaking. It has medium big grain, the portion of sieving fractions above $2.5 \mathrm{~mm}$ is medium high to low. It is resistant to powdery mildew of barley, medium resistant to leaf rust of barley, medium resistant to net blotch of barley, medium resistant to leaf scald, and medium resistant to scab of barley. The yield of sieving fractions above $2.5 \mathrm{~mm}$ in the untreated variant growing in the potato region is very high, in the untreated variant growing in the potato region high, in the treated variant growing in the sugar-beet and cereal regions medium high to high, in both variants growing in the maize region and in the untreated variant growing in the sugar-beet and cereal regions medium high.

\section{Conclusion}

The study presents the results achieved by six varieties of spring barley which were registered in the Czech Republic after the 2018 harvest. The quality was assessed according to the Malting Quality Index. The content of nitrogenous substances in the spring barley varieties under study was at the optimal level (10.2 to $10.6 \%$ ) and the varieties were rich in extract. More than $83 \%$ of extract was recorded in the varieties Laureate (83.9\%), LG Aurus (83.5\%), and Cosmopolitan (83.1\%). Proteolytic, amylolytic and cytolytic modification in the varieties was mostly at the optimal level. The variety Laureate had high Kolbach index (50.6\%). Quality of wort assessed by the apparent final attenuation in the spring barley varieties Cosmopolitan, LG Aurus, Laureate, and Runner was at the optimal level (over 82\%).

\section{Acknowledgements}

The study was funded from the institutional support for long-term strategic development of the research organization provided by the Ministry of Agriculture of the CR (MZE-R01918), project of the Ministry of Education, Youth and Sports of the CR (L01312) and some members of the Czech Beer and Malt Association.

\section{References}

Act No. 219/2003 Coll. on the marketing of seeds and seedlings and amending some other acts. In: Collection of Laws of the Czech Republic. 25 June 2003.

Baxter, E. D., O'Farrell, D. D., 1983: Use of the friabilimeter to assess homogeneity of malt. J. Inst. Brew. (89)3:210-214. https://doi. org/10.1002/j.2050-0416.1983.tb04169.x 
Briggs, D. E., 1998: Malts and Malting. London, Blackie Academic and Professional, ISBN 10: 0412298007

Dvořáčková, O., 2016: Metodika zkoušek užitné hodnoty ječmen. Brno, ÚKZÚZ NOÚ. http://eagri.cz/public/web/ukzuz/portal/odrudy/ registrace-odrud/metodiky-zkousek/

EBC Analysis committee, 2010: Analytica-EBC. Barley: 3.2 Moisture content of barley, 3.3.2 Total nitrogen of barley: Dumas combustion method, Malt: 4.2 Moisture content of malt, 4.3.2 Total nitrogen of malt: Dumas combustion method, 4.5.1 Extract of malt: Congress mash, Soluble nitrogen of malt: Dumas combustion method, 4.10 Free amino nitrogen of malt by spectrophotometry, 4.11 Fermentability, final attenuation of laboratory wort from malt, 4.12 Diastatic power of malt, 4.15 Friability, glassy corns and unmodified grains of malt by friabilimeter, 4.16.2 High molecular weight $\beta$-glucan content of malt: Fluorimetric method, Wort: EBC 8.7 Fermentable Carbohydrates in Wort, Beer: 9.11 Total polyphenols in beer by spectrophotometry, 9.29 Haze in Beer: Calibration of Haze Meters. Nüremberg: Fachverlag Hans Carl, ISBN 978-3-418-00759-5.
European Commission, 2008: Publication of an application pursuant to Article 6(2) of Council Regulation (EC) No 510/2006 on the protection of geographical indications and designations of origin for agricultural products and foodstuffs. Official Journal of the European Union C 16, 23.1.2008, p. 14-22

European Commission, 2019: Common catalogue of varieties of agricultural plant species -37 th complete edition. Official Journal of the European Union C 13, 11.1.2019.

MEBAK, 2011: Raw materials: Barley; Adjuncts; Malt; Hops and Hop Products. 1 Barley: 1.5.3 Micromalting; Malz: 3.1.4.11 Maischmethode nach Hartong-Kretschmer VZ $45{ }^{\circ} \mathrm{C}$. Freising-Weihenstephan, Mitteleuropäische Brautechnische Analysenkommission (MEBAK),

Psota, V., Kosař, K., 2002: Malting Quality Index, Kvasný Průmysl, 47:142148, 2002. https://doi.org/10.18832/kp2002011

Psota, V., Svorad, M., Musilová, M., 2019: Barley Varieties Registered in the Slovak Republic after Harvest 2018. Kvasny Prumysl 65(3), 106-110. 